University of Nebraska - Lincoln

DigitalCommons@University of Nebraska - Lincoln

2-13-2009

\title{
Magnetism of FePt Surface Alloys
}

Jan Honolka

Max-Planck-Institut für Festkörperforschung, honolka@fzu.cz

T. Y. Lee

Max-Planck-Institut für Festko“rperforschung, Heisenbergstrasse 1, 70569 Stuttgart, Germany

K. Kuhnke

Max-Planck-Institut fu“r Festko“rperforschung, Heisenbergstrasse 1, 70569 Stuttgart, Germany

Axel Enders

University of Nebraska-Lincoln, a.enders@me.com

Ralph Skomski

University of Nebraska-Lincoln, rskomski2@unl.edu

See next page for additional authors

Follow this and additional works at: https://digitalcommons.unl.edu/physicsskomski

Part of the Physics Commons

Honolka, Jan; Lee, T. Y.; Kuhnke, K.; Enders, Axel; Skomski, Ralph; Bornemann, S.; Mankovsky, S.; Minar, J.; Staunton, J.; Ebert, H.; Hessler, M.; Fauth, K.; Schutz, G.; Buchsbaum, A.; Schmid, M.; Varga, P.; and Kern, K., "Magnetism of FePt Surface Alloys" (2009). Ralph Skomski Publications. 55.

https://digitalcommons.unl.edu/physicsskomski/55

This Article is brought to you for free and open access by the Research Papers in Physics and Astronomy at DigitalCommons@University of Nebraska - Lincoln. It has been accepted for inclusion in Ralph Skomski Publications by an authorized administrator of DigitalCommons@University of Nebraska - Lincoln. 


\section{Authors}

Jan Honolka, T. Y. Lee, K. Kuhnke, Axel Enders, Ralph Skomski, S. Bornemann, S. Mankovsky, J. Minar, J. Staunton, H. Ebert, M. Hessler, K. Fauth, G. Schutz, A. Buchsbaum, M. Schmid, P. Varga, and K. Kern 


\title{
Magnetism of FePt Surface Alloys
}

\author{
J. Honolka, ${ }^{1, *}$ T. Y. Lee, ${ }^{1}$ K. Kuhnke, ${ }^{1}$ A. Enders, ${ }^{2}$ R. Skomski, ${ }^{2}$ S. Bornemann, ${ }^{3}$ S. Mankovsky, ${ }^{3}$ J. Minár, ${ }^{3}$ J. Staunton, ${ }^{4}$ \\ H. Ebert, ${ }^{3}$ M. Hessler, ${ }^{5}$ K. Fauth, ${ }^{6}$ G. Schütz, ${ }^{5}$ A. Buchsbaum, ${ }^{7}$ M. Schmid, ${ }^{7}$ P. Varga, ${ }^{7}$ and K. Kern ${ }^{1}$ \\ ${ }^{1}$ Max-Planck-Institut für Festkörperforschung, Heisenbergstrasse 1, 70569 Stuttgart, Germany \\ ${ }^{2}$ Department of Physics and Astronomy and NCMN, University of Nebraska, Lincoln, Nebraska 68588, USA \\ ${ }^{3}$ Department Chemie und Biochemie, Ludwig-Maximilians-Universität München, 81377 München, Germany \\ ${ }^{4}$ Department of Physics, University of Warwick, Coventry CV4 7AL, United Kingdom \\ ${ }^{5}$ Max-Planck-Institut für Metallforschung, Heisenbergstrasse 13, 70569 Stuttgart, Germany \\ ${ }^{6}$ Physikalisches Institut, Universität Würzburg, Am Hubland, 97074 Würzburg, Germany \\ ${ }^{7}$ Institut für Allgemeine Physik, Technische Universität Wien, Wiedner Hauptstrasse 8-10/134, A 1040 Wien, Austria
}

(Received 30 July 2008; published 13 February 2009)

\begin{abstract}
The complex correlation of structure and magnetism in highly coercive monoatomic FePt surface alloys is studied using scanning tunneling microscopy, x-ray magnetic circular dichroism, and ab initio theory. Depending on the specific lateral atomic coordination of Fe either hard magnetic properties comparable to that of bulk FePt or complex noncollinear magnetism due to Dzyaloshinski-Moriya interactions are observed. Our calculations confirm the subtle dependence of the magnetic anisotropy and spin alignment on the local coordination and suggest that $3 \mathrm{D}$ stacking of $\mathrm{Fe}$ and Pt layers in bulk $L 1_{0}$ magnets is not essential to achieve high-anisotropy values.
\end{abstract}

DOI: 10.1103/PhysRevLett.102.067207

Several exciting experiments on $\mathrm{Fe}$ atoms in nanostructures have shown recently that the magnetism of lowcoordinated $\mathrm{Fe}$ is very different from bulk, rich in features, with a strong tendency towards nonparallel alignment of neighboring spins. Examples include antiferromagnetism in Fe monolayers on tungsten surfaces [1], and the complex noncollinear spin alignment in Fe monolayers on $\operatorname{Ir}(111)$ [2]. Common to these results is the presence of lowcoordinated $\mathrm{Fe}$ atoms and bond lengths different from bulk Fe, as well as the direct contact between Fe atoms and a $5 d$ element substrate. The complexity of the magnetism in such systems is a result of the quasi-2D interplay between exchange interaction, magnetic anisotropy, induced moments in $5 d$ atoms, and the emergence of Dzyaloshinski-Moriya (DM) [3] interactions due to the broken inversion symmetry. In this work, we succeeded in embedding Fe atoms directly in the surface layer of a $\operatorname{Pt}(997)$ substrate and created a new class of lowdimensional materials in which mechanisms that lead to complex magnetic behavior can be studied in more detail. STM images with atomically resolved chemical contrast are presented and the system's magnetic properties are analyzed by x-ray magnetic circular dichroism (XMCD). We show that depending on the specific lateral coordination of Fe the magnetism of these surface alloys (SA) can exhibit large out-of-plane magnetic anisotropy, comparable to that of high-anisotropy bulklike FePt alloy films (10-100 nm thickness) [4-6], and noncollinear magnetism due to DM interactions. While in bulk FePt large anisotropies are due to noncubic 3D stacking of Fe and Pt layers $[7,8]$, we find a substantially different mechanism for SAs.

FePt SAs are grown on $\mathrm{Pt}(997)$ substrates with a highly regular step periodicity of $2 \mathrm{~nm}$ [9]. FePt alloy layers
PACS numbers: 75.70. $-\mathrm{i}, 75.30 .-\mathrm{m}, 75.50 . \mathrm{Bb}, 75.75 .+\mathrm{a}$

confined to the topmost layer of the $\operatorname{Pt}(997)$ surface form during molecular-beam epitaxy (MBE) deposition of submonolayer coverages of $\mathrm{Fe}$ at temperatures of 500 to $550 \mathrm{~K}$. The substrate and alloy preparation and structural characterization is described in Ref. [10]. STM images shown in Fig. 1 resolve SA structures that form during

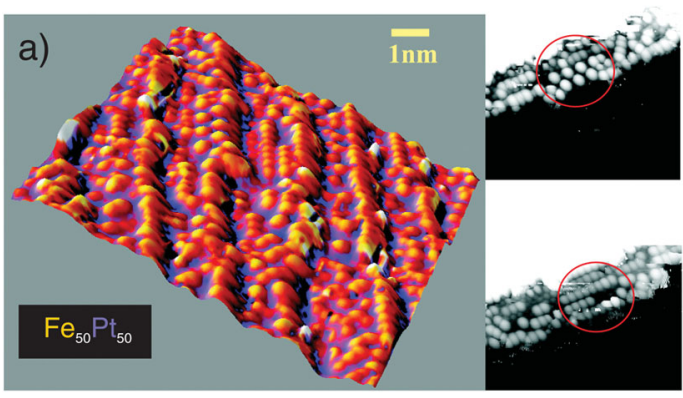

$2 \times 2$ alloy
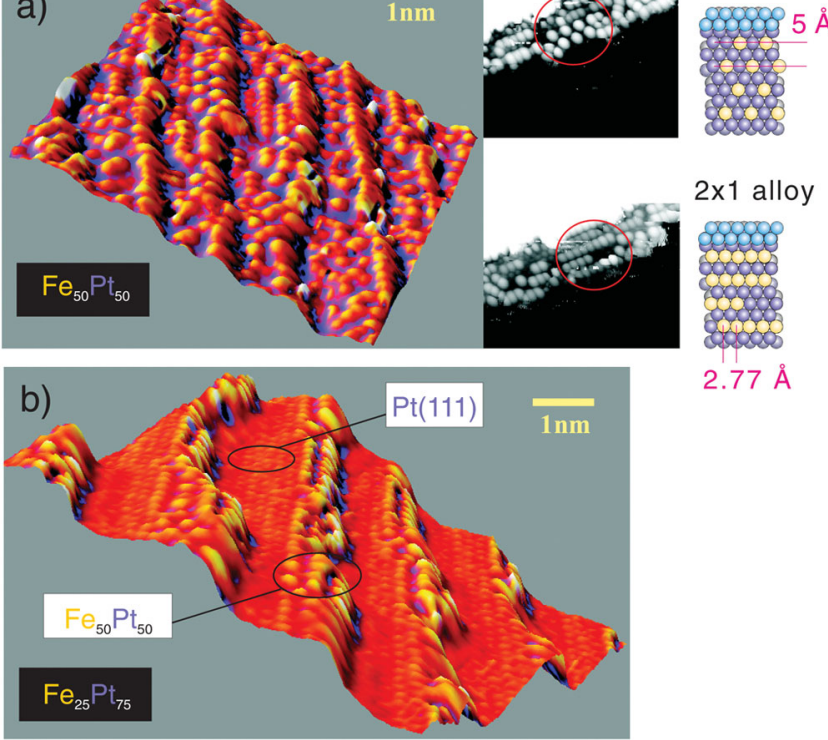

$2.77 \AA$

FIG. 1 (color). STM images of $\mathrm{Fe}_{x} \mathrm{Pt}_{1-x} \mathrm{SAs}$ formed during deposition of $\mathrm{Fe}$ atoms onto $\mathrm{Pt}(997)$ at $525 \mathrm{~K}$. (a) $\mathrm{Fe}_{50} \mathrm{Pt}_{50}$ : Mixture of $\mathrm{Fe}$ and $\mathrm{Pt}$ atoms in the surface layer consistent with the expected stoichiometry. Fe $2 \times 2$ and $2 \times 1$ superstructures on a local scale are clearly visible (right side). (b) $\mathrm{Fe}_{25} \mathrm{Pt}_{75}$ : Partial $\mathrm{Fe}_{50} \mathrm{Pt}_{50} \mathrm{SA}$ with preferential $2 \times 1$ superstructure confined close to the step edges. 
the deposition of Fe at $525 \mathrm{~K}$. The SA grows from the step edges into the terraces; offering less Fe leads to an alloy which is confined to the area around the step edges, as shown in Fig. 1(b) for $\mathrm{Fe}_{25} \mathrm{Pt}_{75}$. Figures 1(a) and 1(b) show equiatomic $\left(\mathrm{Fe}_{50} \mathrm{Pt}_{50}\right)$ and iron-poor $\left(\mathrm{Fe}_{25} \mathrm{Pt}_{75}\right)$ coverages, respectively. The enlarged areas of individual terraces in Fig. 1(a) show typical SA configurations, especially $2 \times 1$ and $2 \times 2$ structures, which are also illustrated in the cartoons of Fig. 1. We observe that the degree of disorder depends on the iron concentration; the $2 \times 1$ and-especially $-2 \times 2$ structures are more predominant for low $\mathrm{Fe}$ coverages. In equiatomic $\mathrm{SAs}\left(\mathrm{Fe}_{50} \mathrm{Pt}_{50}\right)$, shown in Fig. 1(a), the $2 \times 1$ and $2 \times 2$ structures are confined to very limited areas. We find the lateral atomic environments of the Fe and Pt atoms in Fig. 1 to be of crucial importance for the understanding of anisotropy and coercivity.

The in situ magnetic characterization of the FePt SAs was carried out using XMCD measurements [11] at a fixed temperature of $T=12 \mathrm{~K}$ and magnetic fields up to $2.5 \mathrm{~T}$. From the difference between absorption spectra (XAS) for opposite sample magnetization $\vec{M}$ the magnetic moments projected on the vector of the incoming photon spin can be determined [12]. In order to probe the systems magnetic anisotropies we measured the magnetic behavior in different directions parallel and perpendicular to the substrate steps, in the (11) $)$ and $(\overline{1} 10)$ planes, respectively. The polar angle $\theta$ is the angle of incidence of the $\mathrm{x}$ rays with respect to the surface normal, as shown in Fig. 2(a). The upper part of Fig. 2(b) displays typical XAS spectra at the Fe $L_{3,2}$ and Pt $N_{7,6}$ absorption edges of the $\mathrm{Fe}_{50} \mathrm{Pt}_{50}$ surface alloys. The difference in the XAS for opposite magnetic fields (plotted in the lower part as the XMCD signal) reflects a magnetization at both Fe and Pt absorption edges. The Pt exhibits a dichroic signal as a result of an induced magnetic moment

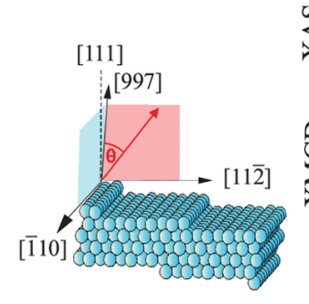

a)

b)

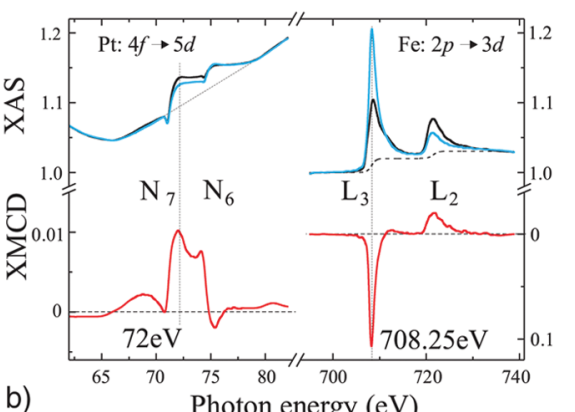

FIG. 2 (color). (a) Geometry: XMCD was measured along different $\operatorname{Pt}(997)$ directions parallel and perpendicular to the substrate steps (within the planes in blue and red, respectively). $\theta$ is the polar angle with respect to the surface normal. (b) XAS of the 0.5 ML FePt SA at the Pt $N_{7,6}$ (left) and Fe $L_{3,2}$ edges (right). The dashed two-step function accounts for the contribution of the $2 p-4 s$ transitions to the Fe XAS. The difference between the signals for opposite magnetization (blue and black traces) yields the XMCD spectra (red traces). The photon energies at which $M(B)$ for Fe and $\mathrm{Pt}$ is measured [Fig. 3(a)] are indicated by vertical lines. due to a strong hybridization between $\mathrm{Fe} 3 d$ and $\mathrm{Pt} 5 d$ states. The complicated Fano line shape at the Pt $N_{7,6}$ edges has been analyzed in detail in an earlier study of bulk $\mathrm{CoPt}_{3}$ [13]. We used the dichroism at the Fe $L_{3}$ and the Pt $N_{7}$ absorption edges at 72.0 and $708.3 \mathrm{eV}$, respectively, to obtain element-specific magnetization curves. Figure 3(a) shows typical hysteresis loops for Fe and Pt along $\theta=0^{\circ}$ and $70^{\circ}$. We observe a nearly square-shaped hysteresis loop along the surface normal $\left(\theta=0^{\circ}\right)$, corresponding to the polar magnetic easy axis (perpendicular anisotropy). The coercive field $B_{c}=0.71 \mathrm{~T}$ along this direction suggests the presence of a large magnetic anisotropy energy (MAE). With increasing polar angle $\theta$, the loops loose their squareness [blue and red curves in Fig. 3(a)].

The $\theta=70^{\circ}$ hysteresis loops exhibit an additional inplane anisotropy, since the two in-plane directions parallel and perpendicular to the step edges yield slightly different coercivities [Fig. 3(a), right]. This symmetry breaking is attributed to the coexistence of ordered $2 \times 2$ and $2 \times 1$ superstructures. Both surface structures support uniaxial MAE $\sim K_{u} \sin ^{2} \bar{\theta}$, and our XMCD experiments reveal easyaxis anisotropy $\left(K_{u}>0\right)(\bar{\theta}$ is the angle between $M$ and the surface normal). However, only the $2 \times 1$, but not the $2 \times$ 2 surface, exhibits a $K_{1}^{\prime}$-type anisotropy [see Eq. (3.2) in Ref. [14]], which favors in-plane spin alignment parallel or perpendicular to the monatomic wires, depending on the sign of $K_{1}^{\prime}$. A similar effect is also expected from the residual influence of the steps.

We find congruent $\mathrm{Pt}$ and $\mathrm{Fe}$ hysteresis loops in Fig. 3(a), suggesting a collinear alignment of Fe and Pt moments. To distinguish between a ferromagnetic (FM) and anti-FM ordering, we observe that the sign of the XMCD signal at the Fe $L_{3,2}$ and $\mathrm{Pt} N_{7,6}$ edges in Fig. 2(b) exhibits the same relation as found by Shishidou et al. for the Co $M_{3,2}$ and $\mathrm{Pt} N_{7}$ edges of the bulk ferromagnet $\mathrm{CoPt}_{3}$ [13]. By analogy, we conclude that in the FePt SA the Pt and
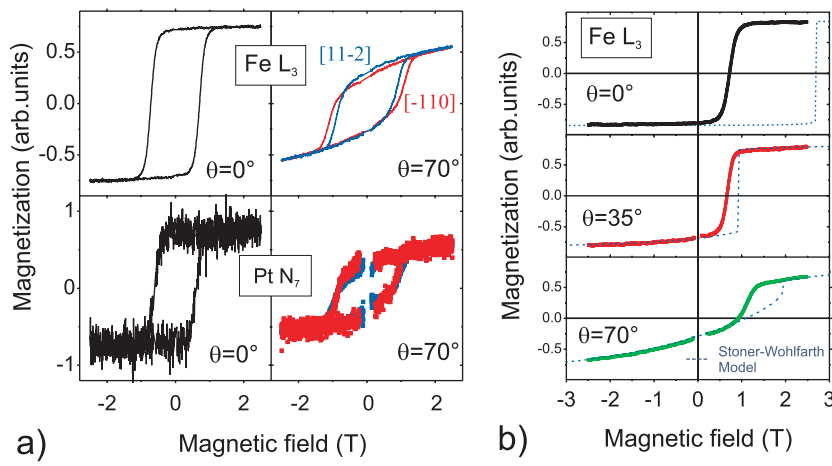

FIG. 3 (color). (a) Upper panels: Hysteresis of the $\mathrm{Fe}_{50} \mathrm{Pt}_{50} \mathrm{SA}$ taken at the $\mathrm{Fe} L_{3}$ edge for directions $\theta=0^{\circ}, 70^{\circ}$. The data are normalized to the nondichroic XAS signal. The measured directions are color coded with orientation labels explained in Fig. 2(a) and in the text. Lower panels: Corresponding measurements at the Pt $N_{7}$ edge. (b) Hysteresis of $\mathrm{Fe}_{50} \mathrm{Pt}_{50}$ for $\theta=0^{\circ}$, $35^{\circ}$ and $70^{\circ}$ together with a Stoner rotation fit using $K_{u}=$ $0.35 \mathrm{meV} /$ f.u. and $m_{\text {f.u. }}=3.53 \mu_{B} /$ f.u. 
Fe moments are FM coupled. Moreover, the relative size of the XMCD signal at the $\mathrm{Pt} N_{7}$ edge suggests that the induced moment of Pt within the alloy layer is comparable to the one found in $\mathrm{CoPt}_{3}$. For the latter, a Pt moment of $0.26 \mu_{B}$ /atom was determined by neutron scattering $[15,16]$.

Our experimental results are supported by fully relativistic $a b$ initio density functional theory (DFT) simulations. The electronic structure of the surface alloys on $\operatorname{Pt}(111)$ was calculated by the KKR multiple scattering formalism, and the local LSDA was used for the exchange and correlation effects. The anisotropy was extracted from calculations of the magnetic torque, and in the investigation of random SAs, the disorder was treated in the coherentpotential approximation. The calculations confirm a parallel alignment of $\mathrm{Fe}$ and $\mathrm{Pt}$ spins, and predict induced $\mathrm{Pt}$ moments of $0.27 \mu_{B}$ per Pt atom in a FePt layer with $2 \times 1$ structure on $\mathrm{Pt}(111)$ and $0.21 \mu_{B}$ per Pt atom for the disordered FePt surface alloy. For the first subsurface layer we obtain Pt moments of about $0.2 \mu_{B}$ and $0.18 \mu_{B}$, respectively. The magnetism of the surface alloy depends on the Fe and the Pt sublattice due to interatomic hybridization, hence it has to be treated as one system. It is best defined as the top $\mathrm{Fe}_{50} \mathrm{Pt}_{50}$ alloy layer plus the Pt layer underneath, giving an overall chemical composition, or formula unit (f.u.), of $\mathrm{FePt}_{3}$.

Using DFT, we systematically calculated various FePt model structures on $\operatorname{Pt}(111)$, as observed in our STM experiments. $\mathrm{Pt}(997)$ steps were simulated by a finite monolayer high cluster of $\mathrm{Pt}$ on $\mathrm{Pt}(111)$. For the Pt-rich $2 \times 2$ structure on $\mathrm{Pt}(111)$, we found an AFM coupling between Fe nearest neighbors, leading inevitably to magnetic frustration due to the hexagonal symmetry of the SA [Fig. 1(a)]. By contrast, the simulation of ideal $2 \times 1$ SA structures yields a strong FM exchange within each $\mathrm{Fe}$ chain of $60 \mathrm{meV}$ between neighbor atoms. The coupling between adjacent $\mathrm{Fe}$ chains, however, is much smaller, about $1 \mathrm{meV}$ per atom. It will be shown below that this weak interchain coupling facilitates the formation of complex spin configurations due to DM interactions. The simulation of random $\mathrm{Fe}_{50} \mathrm{Pt}_{50}$ SAs yields strong FM exchange of about $48 \mathrm{meV}$ between Fe nearest neighbors.

The magnetic hysteresis of nanoscale systems with rigid FM coupling is well-described by the coherent-rotation or Stoner-Wohlfarth model. The calculation of $M(B)$ and the extraction of the anisotropy constant $K_{u}$ requires the knowledge of the magnetic moment per Fe atom, which includes both $\mathrm{Fe}$ and the induced Pt moments. The Fe moment was estimated from the XMCD spectra using the sum rules for $3 d$ metals $[17,18]$. From the saturated spectra along the magnetic easy axis we derive an effective spin moment per hole, $m_{S}^{\text {eff }}=m_{S}+7 m_{T}$, of $0.62 \mu_{B} /$ hole, where $m_{T}$ is the magnetic dipole term accounting for the anisotropy of the spin density. Because of the influence of $m_{T}$ the actual spin moment $m_{S}$ per hole in the polar direction is expected to be approximately $15 \%-20 \%$ larger than the experimentally determined $m_{S}^{\text {eff }}$. Under the assumption of $3.7 d$-band holes per atom [19], the actual spin moment per Fe atom is $2.75 \mu_{B}$, which is fairly close to the DFT prediction $\left(m_{S}=3.2 \mu_{B}\right)$. Taking the experimental Fe moment and adding three Pt neighbors with $\mu_{\mathrm{Pt}}=0.26 \mu_{B}$ each, we obtain a total moment of $3.53 \mu_{B}$ per $\mathrm{FePt}_{3}$ formula unit (or $\mathrm{Fe}$ atom).

Figure 3(b) compares our $M(B)$ data at $\theta=0^{\circ}, 35^{\circ}$ and $70^{\circ}$ with the Stoner-Wohlfarth model. Best agreement is achieved for an effective magnetic anisotropy constant $K_{u}=0.35 \mathrm{meV}$ per Fe atom. This anisotropy is comparable to that of highly anisotropic $L 1_{0}$ FePt bulk alloys, $K_{u}=5 \mathrm{MJ} / \mathrm{m}^{3}\left(5 \times 10^{7} \mathrm{erg} / \mathrm{cm}^{3}\right)$ [20], or $0.8 \mathrm{meV}$ per Fe atom (In terms of anisotropy fields, $1 \mathrm{meV} / \mu_{B}=$ $34.6 \mathrm{~T})$. The strong spin-orbit coupling of $\mathrm{Pt}, \xi_{\mathrm{Pt}}=$ $0.6 \mathrm{eV}$, compared to $\mathrm{Fe}, \xi_{\mathrm{Fe}}=0.08 \mathrm{eV}$, is known to significantly contribute to the anisotropy. Figure 3 shows that the Stoner-Wohlfarth model works well in the coherentrotation regime, that is, at larger fields, and adds evidence to the existence of a magnetically stable monatomic $\mathrm{Fe}_{50} \mathrm{Pt}_{50}$ alloy layer. The much less perfect agreement in small field, especially for $\theta=0^{\circ}$, is a common phenomenon caused by real-structure imperfections [14] and, in the present case, by the neglect of magnetostatic interactions. Both effects reduce the coercivity.

The magnetization loops exhibit a delicate dependence on the details of the Fe-Pt coordination. Figure 4 compares the polar magnetization curve of the $\mathrm{Fe}_{50} \mathrm{Pt}_{50} \mathrm{SA}$ in (a) with loops of a SA layer formed from only 0.35 ML Fe at $525 \mathrm{~K}$ (b), and with nonalloyed Fe stripes of 4 atoms' width formed from 0.5 ML Fe at $350 \mathrm{~K}$ (c). Figure 4(b) shows that confining the alloy to a region close to the step edges drastically alters the loop shape. Compared to the square loop of the $\mathrm{Fe}_{50} \mathrm{Pt}_{50}$ alloy, the loop in (b) is $S$ shaped, and remanence and coercivity are reduced by $78 \%$ and $68 \%$, respectively. The overall shape of the loop (b) is very similar to that of the nonalloyed Fe stripes of $0.5 \mathrm{ML}$ $\mathrm{Fe}$ (c). Note that the available field of $2.5 \mathrm{~T}$ is insufficient

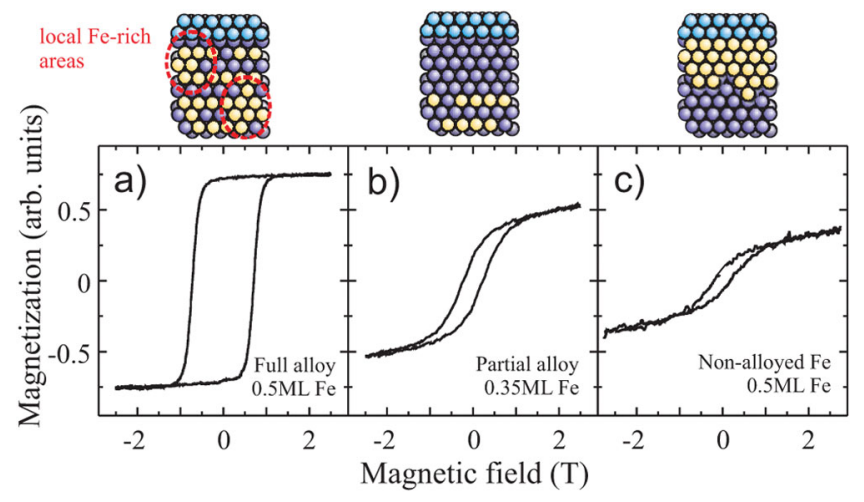

FIG. 4 (color). $\quad M(B)$ perpendicular to the surface at the Fe $L_{3}$ edge of (a) the FePt SA prepared with $0.5 \mathrm{ML} \mathrm{Fe,} \mathrm{(b)} \mathrm{a} \mathrm{SA}$ prepared with $30 \%$ less Fe, and (c) a nonalloyed Fe-stripe with $0.5 \mathrm{ML}$ Fe. All three samples can be shown to exhibit a perpendicular easy magnetization axis. 
to saturate the magnetization, however, full saturation has been achieved at $B=6 \mathrm{~T}$ on nonalloyed $0.5 \mathrm{ML} \mathrm{Fe}$ on $\mathrm{Pt}(997)$ in separate experiments at a different beam line. What makes $\mathrm{Fe}_{50} \mathrm{Pt}_{50}$ surface alloys such hard magnets? High coercivity and slow saturation require high magnetocrystalline anisotropy and strong exchange coupling. In $L 1_{0}$-ordered bulk FePt, the strong $\mathrm{Fe}-\mathrm{Fe}$ intralayer exchange provides the basis for magnetic order and ferromagnetism. Our experiments and the $a b$ initio theoretical results show that the situation in two-dimensional FePt is more complex. Here, strong out-of-plane anisotropy is found for equiatomic FePt coverages (0.5-0.6 ML Fe) where the area of the ordered regions is substantially decreased. The DFT simulation of the statistically disordered $\mathrm{Fe}_{50} \mathrm{Pt}_{50}$ SA yields a weak in-plane anisotropy of $0.09 \mathrm{meV}$ per $\mathrm{Fe}$ atom, while a full $\mathrm{Fe}$ monolayer has a weak out-of-plane anisotropy of $0.03 \mathrm{meV}$ per Fe atom. By contrast, for a perfect $2 \times 1$ structure, our calculations yield a very large in-plane MAE of $1.06 \mathrm{meV} /$ f.u.. Intriguingly for this system DM [3] interactions play an important role. The calculated DM coupling vector $\vec{D}_{i j}$ between two closest magnetic moments of $\mathrm{Fe}$ in different chains is of the same order of magnitude as the ordinary exchange coupling $\left(\left|\vec{D}_{i j}\right|=4.6 \mathrm{meV} ; J=8.8 \mathrm{meV}\right)$. This leads to the creation of a noncollinear magnetic structure with coupled in-plane and out-of-plane components of the total magnetic moment. In this case the discussion of magnetic anisotropy on the basis of $M(B)$ becomes difficult: for instance, when in- and out-of-plane anisotropies for individual sites have similar magnitudes it will result in similar magnetization curves that cannot be distinguished in a simple way. This effect is particularly pronounced for the system with $0.35 \mathrm{ML}$ Fe coverage [Figs. 1(b) and 4(b)] as it consists of almost perfect stripes of the Fe-Pt $2 \times 1$ alloy and it offers a convincing explanation of the out-of plane magnetization component and of the lower magnetization at $2.5 \mathrm{~T}$ compared to $\mathrm{Fe}_{50} \mathrm{Pt}_{50}$.

As the Fe coverage is increased to about $0.5-0.6 \mathrm{ML}$ [Figs. 1(a) and 4(a)], $2 \times 1$ stripes are destroyed by the substitution of $\mathrm{Fe}$ into the $\mathrm{Pt}$ chains. This results in the formation of small Fe-rich surface regions as those sketched in Fig. 4(a) with lateral Fe-Pt interfaces. Our calculations show that these islands of strongly exchange coupled $\mathrm{Fe}$ atoms exhibit a pronounced out-of-plane anisotropy of $0.3-0.5 \mathrm{meV}$ per $\mathrm{Fe}$ atom. Without the Fe-rich areas, that is, for a $\mathrm{Fe}$ concentration just below 50\% of a full monolayer, the anisotropy is small, as discussed earlier. A slight increase of the Fe concentration beyond $50 \%-60 \%$ results also in a significant decrease of the anisotropy, and for a Pt-rich $2 \times 2$ structure $\left(\mathrm{Fe}_{65} \mathrm{Pt}_{35}\right)$, the calculated anisotropy is only $0.15 \mathrm{meV}$ per $\mathrm{Fe}$ atom. Thus, FePt SAs containing 0.5-0.6 ML of iron seem to hit the "sweet spot" for strongest out-of plane anisotropy.

In conclusion, the key result of this paper is in the identification of structural and magnetic phases in $\mathrm{FePt}$ surface alloys, depending on the Fe concentration, and the complex mechanism that leads to a maximum in the magnetic anisotropy for a Fe concentration of 50\%-60\%. The FePt $2 \times 1$ stripe phase exhibits an intriguing interplay between exchange and DM interaction, which results in noncollinear spin structures that appear in the experiments in the form of reduced anisotropy and total moment. Our work, thus, extends recent studies on noncollinear spinstructures of full $\mathrm{Fe}$ monolayers on other $5 d$ transition metal surfaces $[1,2]$ to surface alloys. Strong out-of-plane anisotropy comparable to bulk $L 1_{0}$ alloys are achieved for some surface alloy structures. Both bulk $L 1_{0}$ and surface alloy exploit the strong spin-orbit coupling of Pt, but the anisotropy is realized differently in the two structures, and the surface anisotropy exhibits a more complex physics.

This research is supported by DFG (SPP 1153), NSF CAREER (DMR-0747704), and NSF MRSEC (DMR0213808).

*j.honolka@fkf.mpg.de

[1] M. Bode et al., Nature Mater. 5, 477 (2006).

[2] K. von Bergmann et al., Phys. Rev. Lett. 96, 167203 (2006).

[3] K.-H. Fischer and A. J. Hertz, Spin Glasses (University Press, Cambridge, England, 1991).

[4] M. L. Yan, H. Zeng, N. Powers, and D. J. Sellmyer, J. Appl. Phys. 91, 8471 (2002).

[5] H. Kanazawa, G. Lauhoff, and T. Suzuki, J. Appl. Phys. 87, 6143 (2000).

[6] S. Okamoto et al., Phys. Rev. B 67, 094422 (2003).

[7] S. D. Willoughby et al., J. Appl. Phys. 91, 8822 (2002).

[8] P. Ravindran et al., Phys. Rev. B 63, 144409 (2001).

[9] E. Hahn, H. Schief, V. Marsico, A. Fricke, and K. Kern, Phys. Rev. Lett. 72, 3378 (1994).

[10] T. Y. Lee, S. Sarbach, K. Kuhnke, and K. Kern, Surf. Sci. 600, 3266 (2006).

[11] Experiments were performed at BESSY II (PM-3) in the TEY mode. $B$ is applied collinear to the $\mathrm{X}$-ray beam.

[12] W. L. O'Brien and B. P. Tonner, Phys. Rev. B 50, 12672 (1994).

[13] T. Shishidou et al., Phys. Rev. B 55, 3749 (1997).

[14] R. Skomski, J. Phys. Condens. Matter 15, R841 (2003).

[15] F. Menzinger and A. Paoletti, Phys. Rev. 143, 365 (1966).

[16] A quantitative evaluation of the Pt moments based on the XMCD data is complicated by the unknown fraction of moment-carrying $\mathrm{Pt}$ atoms, in contrast to the $\mathrm{Pt}$ atoms in bulk $\mathrm{CoPt}_{3}$, which all occupy $3 c$ sites in the $L 1_{2}$ structure and have a common moment.

[17] B. T. Thole, P. Carra, F. Sette, and G. van der Laan, Phys. Rev. Lett. 68, 1943 (1992).

[18] P. Carra, B. T. Thole, M. Altarelli, and X. Wang, Phys. Rev. Lett. 70, 694 (1993).

[19] I. Galanakis, M. Alouani, and H. Dreyssé, J. Magn. Magn. Mater. 242, 27 (2002).

[20] T. Suzuki, H. Kanazawa, and A. Sakuma, IEEE Trans. Magn. 38, 2794 (2002). 